\title{
A review on remedies used in treatment of varicose veins and varicocele
}

\author{
Girish D Dahikar *, Dipika D Giradkar, Shagufta A Khan and Rajendra 0 Ganjiwale \\ Institute of Pharmaceutical Education and Research, Borgaon (Meghe), Wardha-442001, Maharashtra, India.
}

GSC Biological and Pharmaceutical Sciences, 2022, 18(02), 244-252

Publication history: Received on 14 January 2022; revised on 17 February 2022; accepted on 19 February 2022

Article DOI: https://doi.org/10.30574/gscbps.2022.18.2.0078

\begin{abstract}
Varicose vein is clinical class of the (CVD) i.e. chronic venous disease, also called as the varicosities. Varicose veins are enlarged, swollen and twisting veins often appearing blue or dark purple. When valves in the veins do not work properly, the blood does not flow effectively. The expansion of varicose veins is often caused by a weakening of valves and walls.

Generally varicose vein is found in females especially in case of pregnancy. Varicose veins generally found in lower extremity, leg and the epididymis. Epididymis is the highly convoluted duct behind the testis along which sperm passes to the vas deferens.

A varicocele is an enlargement of the veins within the loose bag of skin that holds your testicles or scrotum. A varicocele is similar to a varicose vein you might see in your leg. Varicoceles are a common cause of low sperm production and decreased sperm quality, which can cause infertility.
\end{abstract}

The aim of writing this review is to provide information about the varicose vein and varicocele the remedy to be used in its treatment and different tests available for its diagnosis.

Keywords: Varicose vein; Varicocele; Diagnostic tests; Phlebectomy; Sclerotherapy

\section{Introduction}

According to the CEAP (Clinical, Etiological, Anatomical and Pathological Elements), varicose vein is the clinical class of chronic venous disease $[1,2]$.

Varicose veins are common venous disease of the lower extremity which affects more than $33 \%$ of adult's population. Varicose veins are lengthened or broaden expanded and convoluted veins. Varicocele is the mass of varicose veins in the spermatic cord.

A varicocele is an abnormal dilation and cured, flexuous, involute, mazy, sinuous, snaky, vermiculate, zigzag veins of the convoluted and like a tendril uses of a venous plexus associated with the spermatic or ovarian veins that drain the testis.

Varicocele occurs within the spermatic cord and can be palpated on physical examination. Varicose veins and varicoceles both are detected on a physical examination in the erect position.

Various allopathic, ayurvedic and homeopathic medications are available for its treatment although they can't completely cure it [3-5].

\footnotetext{
* Corresponding author: Girish D Dahikar

Institute of Pharmaceutical Education and Research, Borgaon (Meghe), Wardha-442001, Maharashtra, India.

Copyright $(2022$ Author(s) retain the copyright of this article. This article is published under the terms of the Creative Commons Attribution Liscense 4.0.
} 


\subsection{Types of varicose vein}

Different types of varicose veins present in legs and varicocele.

- Truncal varicose veins

- Varicosities of extremities

- Reticular varicose veins

- $\quad$ Side branch varicose vein

- Telangiectasia varicose vein

- $\quad$ Spider vein

\subsection{Sign and symptoms of varicose veins and varicocele}

- Well known dark blue vessels, especially in the legs and feet.

- Continuous pain or devotion along the course of vein.

- $\quad$ Easily tired legs.

- Heaviness in the affected area

- $\quad$ Swelling in the affected area

- Darkening of the skin

- Numbness in the legs or scrotum

- Itching or irritating rash in the legs or scrotum

- Burning sensations.

- Night cramps.

- Pigmentation.

- Exercise intolerance.

- Bulging, rope-like, bluish veins indicate superficial varicose veins.

- Aching and heaviness in a limb, sometimes with swelling, but without any prominent or visible blue veins, may signal a deep varicose vein.

- $\quad$ Discolored, peeling skin, skin ulcers and constant rather than intermittent pain are signs of severe varicose veins.

- $\quad$ Subjective symptoms usually are more severe early in the progression of the disease, less severe in the middle phases, and worse again with advancing age.

- Common symptoms of telangiectasia include burning, swelling, throbbling, cramping and leg fatigue. Pain associated with varicocele usually is a dull ache that is worse after prolonged standing

- $\quad$ Pain and other symptoms may worsen with the menstrual cycle, with pregnancy and in response to exogenous hormonal therapy.

\subsection{Risk factors}

There are a number of risk factors that can increase your likelihood of developing varicose veins and varicocele, including:

- Gender

- Obesity

- Genetics

- Age

- $\quad$ Being Overweight

- Occupation

- $\quad$ Being Pregnant

These risk factors are discussed in more detail below. 


\subsubsection{Gender}

Women are more likely to be affected by varicose veins than men. Research suggests that this may be because female hormones tend to relax the walls of veins, making the valves more prone to leaking. Hormones are chemicals that are produced by the body. In men varicocele may develop in adolescence.

\subsubsection{Genetics}

Your risk of developing varicose veins is increased if a close family member has the condition. This suggests that varicose veins may be partly caused by your genes (the units of genetic material that you inherit from your parents).

\subsubsection{Age}

As you get older, your veins start to lose their elasticity and the valves inside them stop working as well.

\subsubsection{Being overweight}

Being severely overweight puts extra pressure on your veins, which means they have to work harder to send the blood back to your heart. This can put increased pressure on the valves, making them more prone to leaking.

\subsubsection{Occupation}

Some research suggests that jobs that require long periods of standing may increase your risk of getting varicose veins and varicocele. This is because your blood does not flow as easily when you are standing for long periods of time.

\subsubsection{Pregnancy}

When a woman is pregnant, the amount of blood in her body increases to help support the developing baby. This puts extra strain on your circulatory system. Increased hormone levels during pregnancy also cause the muscular walls of the blood vessels to relax. Both of these factors may increase your risk of developing varicose veins.

Varicose veins may also develop during pregnancy as the womb (uterus) begins to grow. As the womb expands it puts pressure on the veins in your pelvic area, which can sometimes cause them to become varicose.

Although being pregnant can increase your risk of developing varicose veins, most women find that the condition significantly improves after their pregnancy [6].

\subsection{Complications in varicose vein and varicocele}

\subsubsection{Complications in varicose vein}

- $\quad$ Phlebitis (inflammation)

- Blood clots (actually in very rare complications)

- Varicose eczema

- $\quad$ Bleeding and venous ulcers

- Deep vein thrombosis

\subsubsection{Complications in varicocele}

- Infertility or low fertility

- Low testosterone

- A shrinking testicle also called testicular atrophy.

\subsection{Diagnosis}

The diagnosis of the varicose veins by using clinical and duplex scanning examination and also by the normal arterial examination. Varicoceles is also diagnosed by physical examination which is the standard diagnostic method for varicocele. The estimation of the presence of varicocele is generally difficult to estimate by using physical examination. Therefore, most of the varicoceles are impalpable asymptomatic and diagnosed by using ultrasound evaluation [7]. 


\subsection{Techniques of test}

- Trendelenburg test

- Perthes test

- Doppler auscultation

\subsubsection{Trendelenburg test}

The test is named for Friedrich Trendelenburg who described it in 1891.

The Trendelenburg test or Brodie-Trendelenburg test is a test which can be carried out as part of a physical examination to determine the competency of the valves in the superficial and deep veins of the legs in patients with varicose vein.

\section{Procedure}

With the patient in the supine position the leg is flexed at the hip and raised above heart level. The veins will empty due to gravity or with the assistance of the examiners hand squeezing blood towards the heart.

A tourniquet is then applied around the upper thigh to compose the superficial veins. But not too tight as to occlude the deeper veins, the leg is then lowered by asking the patient to stand.

Normally the superficial saphenous vein will fill from below within 30-35 seconds as blood from the capillary beds reaches the veins if the superficial veins fill more rapidly with the tourniquet in place there is valvular incompetence below the level of the tourniquet in the "deep" or "Communicating" veins. After 20 seconds if there has been no rapid filling the tourniquet is released if there is sudden filling at this point it indicates that the deep and communicating veins are competent but the superficial veins are incompetent.

The test is reported in two parts, the initial standing up of the patient (positive or negative based on rapid filling) and the $2^{\text {nd }}$ phase ones the tourniquet is removed (positive and negative based upon rapid filling).

For example, a possible outcomes test would be negative - positive meaning that the initial phase of the test was negative indicating competence in the deep and communicating veins and the second phase of the test was positive meaning that there is superficial vein incompetence.

The test can be repeated with the tourniquet at different levels to further pinpoint the level of valvular incompetence.

- $\quad$ Above the knee -to assess the mid-thigh perforters.

- below the knee- to assess incompetence between the short saphenous veins and the popliteal vein.

Superficial veins of the leg normally empty into deep veins, however retrograde filling occurs when valves are incompetent, leading to varicose veins.

\subsubsection{Perthes test}

The perthes test is a clinical test for assessing the potency of the deep femoral vein prior to varicose vein surgery. It is named after German surgeon Georg perthes.

The limb is elevated and an elastic bandage is applied firmly from the toes to the upper $1 / 3$ of the thigh to obliterate the superficial veins only, with the bandage applied the patient is asked to walk for 5 minutes. If deep system is competent, the blood will go through and back to the heart. If the deep system is incompetent, the patient will feel pain in the leg.

This test is sometimes referred to as the Delbet-Mocquot test, named after French physicians Pierre Delbet and Pierre Mocquot.

\subsubsection{Modified perthes test}

The test is done by applying a tourniquet at the level of the sapheno-femoral junction to occlude the superficial pathway, and then the patient asked to move in situ. If the deep veins are occluded, the dilated veins increase in prominence.

This is a more objective test as it does not depend on patient's pain threshold. 


\subsubsection{Doppler auscultation test}

Doppler examination is an adjunct to the physical examination that can directly show whether flow in a suspect vein is antegrade, retrograde or to and fro [8].

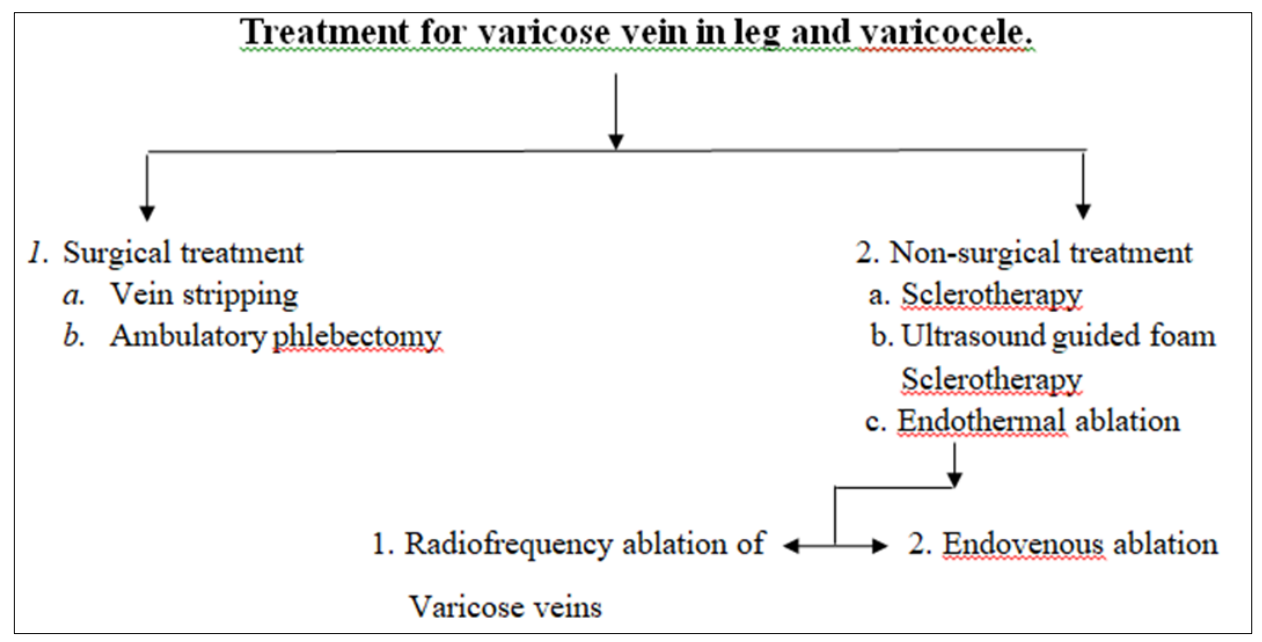

Figure 1 Treatment options for varicose vein and varicocele

\subsection{Remedy used for the treatment of varicose vein and varicocele}

Various allopathic, ayurvedic and homeopathic medications are available for its treatment although they can't completely cure it.

Allopathic remedy for varicose vein and varicocele.

\subsubsection{Methylhesperidin (cadisper-c)}

Methylhesperidin is a plant chemical that is classified as a bioflavonoid. Methylhesperidin is most commonly used for blood vessel condition such as hemorrhoids, varicose veins and poor circulation (venous stasis).

\subsubsection{Morrhuate sodium injection}

Morrhuate sodium injection, USP. This medication is a mixture of saturated and unsaturated fatty acids of cod liver oil. Prescribe for varicose veins is prepared by the saponification of selected cod liver oil morrhuate sodium injection is used for obliteration of primary varicose veins that consist of simple dilation with competent valves.

\subsubsection{Polidocanol injection}

Polidocanol is used as a sclerolant irritant injected to treat varicose veins under the trade names Asclera, Aethoxysklerol and Varithena. Polidocanol causes fibrosis inside varicose veins, occluding the lumen of the vessel, and reducing the appearance of the varicosity. The FDA has approved polidocanol injections for the treatment of small varicose (less than $1 \mathrm{~mm}$ in diameter) and reticular vein (1 to $3 \mathrm{~mm}$ in diameter). Polidocanol works by damaging the cells lining of blood vessels, causing them to close and eventually be replaced by other types of tissue $[9,10]$.

\subsubsection{Rutin (Artisol)}

Rutin is plant pigment (flavonoid) that is found in certain fruits and vegetables. Some peoples believe that rutin can strengthen blood vessels so they use it for varicose vein internal bleeding hemorrhoids and to prevent strokes due to broken veins of arteries.

\subsubsection{Sodium tetradecyl sulfate}

Sodium tetradecyl sulfate used to treat small uncomplicated varicose veins in the legs, if patient in allergic then he should not resive sodium tetradecyl sulfate. Sodium tetradecyl sulfate is a sclerosing agent it works by increasing the formation of blood clots and scar tissue inside certain types of veins. This helps decrease dilation of enlarge veins. Sodium tetradecyl sulfate is use to treat small uncomplicated varicose veins in the leg. It is also use in case of varicocele [11]. 


\subsection{Ayurvedic remedy for varicose vein and varicocele}

\subsubsection{Horse chestnut seed extract}

Horse chestnut seed extract were used for the management of the chronic venous insufficiencies in Europe and Germany. Crude or unprocessed seeds are not used because it may prove to be noxious to health. Contents of the horse chestnut seed extract include aescin, tannins, flavanoids, quinines, sterols and some fatty acids, coumarins and scopolin, of which aescin is the most active constituent of the horse chestnut seeds and comprises about 16-20\%. The vascular permeability of the veins and capillaries is treated by this active constituent aescin. It acts by decreasing the hyper permeability of the veins and also decreases the inflammation and edema which results in improved venous pressure and blood flow. Besides, the extracts also have the antioxidant properties which help in toning the veins, reduce the vascular permeability and enhance the venous return. The hydroxyl coumarin has the anti-thrombin action on to the veins. Many oral and topical formulations have been utilized.

\subsubsection{Gotu kola (Centella asiatica)}

The drug Gotu kola is known for its use in the treatment of skin disorders and it also promotes healthy veins. As per the Indian Pharmacopoeia of the $19^{\text {th }}$ century it has the ability to cure the various skin diseases such as leprosy, varicose ulcers, psoriasis, eczema, etc. Asiaticoside is the active constituent of the plant in which a triglyceride moiety is linked with the aglycone moiety, asiatic acid. It possesses antioxidant, antiulcer and anti-inflammatory activity. Madecassol is a triterpenoid derivative obtained from the plant. The triterpenoids such as saponins and sapogenins shows the wound healing properties and have vascular effects. The activity is because it increases the collagen formation at the site of injury (wound). Hypertension can be reduced by the compound Centelloside and its derivatives. These constituents work collectively to strengthen the walls of the blood vessels and enhance the blood flow through the veins. The permeability of the veins is increases by hyperplasia which is provoked by the active constituents of the plant. Besides the above dosage forms, gotu kola when taken in the form of tea by adding its leaves into the boiling water, it provides relief from the symptoms of the varicose veins and also helps in its treatment [12].

\subsubsection{Apple cider vinegar}

An experiment conducted on a certain group of patients suffering from varicose veins revealed that the application of apple cider vinegar helped in providing the relief from the pain, edema, irritation, pigmentation, ulceration, fatigue, and other symptoms of the disease. The patients were asked to apply the apple cider vinegar on the affected region and keep it covered using a cloth for about 30 minutes twice a day for a month along with the doctor's treatment. On the completion of the test, the statistical data showed that there was decrease in the pain, fatigue, edema, itching, pigmentation, and cramps as compared to the patients who were not asked to apply the vinegar. Acetic acid is the most important component of the apple cider vinegar. Other components include pectin, polyphenols, carotenoids. These constituents have the prebiotic and antibacterial activity which results in the health benefits. Thus, it was concluded that the harmonizing function of apple cider vinegar increases the effect of other routine treatments. No reliable data was found regarding the dose of this drug [13,14].

\subsubsection{Butcher's broom (Ruscus aculeatus)}

The plant contains steroidal saponins, ruscogenin, and neoruscogenin as the active constituents. Other chemical compounds comprise of steroidal saponins, sapogenins, sterols, triterpenes, coumarins, flavanoids, glycolic acid, sparteine, tyramine, etc. Roots of the plant contains higher amount of ruscogenins, hence have been used conventionally for the therapeutic purpose. The in vivo and in vitro studies give an idea about the vaso-constrictive effect of the plant extract and its ability to reduce the vascular permeability. These activities clarify the use of the herb in patients with interminable venous deficiency. Studies state that the plant's activity takes place due to one of the following reasons; activation of post junctional alpha- 1 and alpha-2 adrenergic receptor activation or the alpha-adrenergic obstruction. A study also uncovers that the vaso-constrictive effect may be a result of histamine induced permeability intervened by calcium and alpha-1 adrenergic receptors. The clinical trials; placebo-controlled trials and the open trials showed improvement in the conditions resulting from varicose veins and provided relief from its symptoms such as itching, edema, cramping, and strain in the legs [15].

\subsubsection{Garlic}

Garlic has been used as a customary drug for treating various diseases because of its different medicinal applications and health advantages. The chemical constituents of Garlic include- allicin, allin, ajoene, dially polysufides, Sallycysteine, saponins, glycosides, amino acids, vitamins and minerals. It helps reducing the blood pressure and also possesses antioxidant properties. Apart from this, it is also said to have protein breakdown ability which distributes the protein substance evenly in the body and hence results in increased supply of protein to the lower limbs [16]. 


\subsubsection{Emblica officianalis}

The plant known as Amla, or Indian gooseberry is an important Ayurvedic medicine having enormously high health related benefits. It is rich in Vitamin $\mathrm{C}$, iron and calcium, hence has a powerful antioxidant or free radical scavenging activity which provides protection to the skin. This helps prevention of ulcers and other possible infections. It is also capable of controlling high blood pressures and thus treating the associated complications. The low molecular weight hydrolysable tannins are responsible for protection from the destructive effects of the free radicals by recyclisation of the sugar moieties. This activity of Amla is dependent upon the chemical constituents such as emblicanin A and B, punigluconin and pedunculagin. It is also said to have anti-inflammatory action again due to the presence of high amount of ascorbic acid. Vitamin C helps in improving the fragility of the capillaries. In this way, the varicose veins and its symptoms may be treated by intake of Amla along with the regular diet [17-19].

\subsubsection{Tomato}

Tomatoes are known for their main chemical constitutes Lycopene and $\beta$-carotene. It has a crucial antioxidant property resulting from the free radical quenching action of the lycopene. It neutralizes the action of the free radicals that are very unsafe to the health. Moreover, presence of vitamin $C$ and pro vitamin $A$ enhances the action of lycopene and $\beta$ caroten. It also helps in vasodilation of the constricted blood vessels that is caused due to the high blood pressure and other related causes. The wounds can also be repaired and healed as the chemical moieties promote the development of connective tissues that helps at the time of recuperating of the wound from the body. The flavonoids are beneficial in strengthening the walls of the blood vessels, all contributing towards treatment of varicose veins and allied symptoms $[20,21]$.

\subsubsection{Grape seed extract}

The extract consists of a polyphenolic compound, known as proanthcyanidin. The compound is obtained from the seeds of the red grape and is chemically regarded as oligomeric flavonoid. Proanthocyanidin is said to have anti-oxidant, antiinflammatory and vasodilating activity. The drug helps in dilating the constricted blood vessels, capillary permeability and reducing the blood pressure to the normal range. The effects of proanthocyanidin were evaluated and it was claimed that the drug was capable of reducing the itching, heaviness, and pain. It was also found to be helpful in reducing the swelling caused due to varicose veins.

\subsubsection{Citrus fruits}

The citrus fruits majorly include Oranges, Sweet lime, and Lemon. The major constituents of citrus fruits are vitamin C, flavanoids, dietary fibers, folic acid, etc. These components show anti-oxidant properties similar to Tomatoes and Amla. These fruits have the similar action of quenching the free radicals and preventing their harmful effects. It also helps in lowering the blood pressure and increasing the proportions of High density lipoprotein (good cholesterol). This prevents the narrowing of the blood vessels and thus promotes normal flow of blood through the vessels. Other than this, some studies also suggest that the use of Hespiridin (a flavanoid obtained from orange peels) in combination with Diosmin may be an effective treatment option because of its anti-inflammatory, anti-oxidant and anti-allergic action which helps in reducing the swelling, ulcers, bleeding, cramps, etc. Therefore, helps in treatment of varicose veins.

\subsubsection{Rubia cardifolia}

The dried powder of the stem and root of the plant Rubia cardifolia commonly known as Indian Madder or Manjistha in hindi is used as the effective blood purifier. The drug is traditionally known for its detoxifying action which helps in purification and thinning of the blood. In addition, the drug also shows anti-inflammatory and anti-oxidant action which soothes the toxic effects of varicose veins [21-27].

\subsection{Homeopathic remedy for varicose vein and varicocele}

\subsubsection{Hamamelis}

Hamamelis virginiana, known as American witch -hazel, is a species of witch-hazel native to eastern north America, from nova scotia west to Minnesota, and south to central Florida to eastern. It is one of best homeopathic remedies for varicose veins, where is feeling of tiredness in the legs and a sore, bruised feeling in the muscles of the leg. The vein may bleed and be inflamed. 


\subsubsection{Pulsatilla}

It is one of best homeopathic medicines for varicose veins during pregnancy, there is intense pain in the veins generally the patient is usually thirstless, feels better in open air and unable to tolerate heat or warm weather.

\subsubsection{Calcarea fluorica}

The Calcarea fluorica homeopathic remedy is derived from the original chemical compound of fluoric acid and lime.

\subsubsection{Graphites}

It is one of best homeopathic medicine in cases where there is itching of veins. It is also used in cramping pains in the legs, generally the patients is usually obese, constipated and may have other skin problems.

\subsubsection{Arnica}

One of best homeopathic remedy for the sore, bruised sensation in the varicose vein and there is feeling as if one has been beaten the bluish or blackish discoloration of the veins may be presented, any exertion aggravates the trouble.

\subsubsection{Lachesis}

It is used in treatment of bluish- purple-colored veins, especially if they appeared in menopause [28,29].

\section{Conclusion}

So many patients suffering from the varicose vein and varicocele usually have to undergoes through various complex treatment, surgical and non- surgical they are include so many convoluted processes and number of complications. Varicose vein affects more than $30 \%$ adults population and varicocele affects $11-15 \%$ adults men. Aim of this article is to provide the complete information about the remedy which is use in treatment of varicose vein and varicocele. These drugs not only assure an enduring effect on the venous disease but also they prove to be cost effective and it is help to the patients for the recovery.

\section{Compliance with ethical standards}

\section{Acknowledgments}

The authors are thankful to the Principal of Institute of Pharmaceutical Education and Research, Borgaon (Meghe), Wardha and Management of the institute VYWS, Amravati, Maharashtra, for their contribution to see that this manuscript comes to a success.

\section{Disclosure of conflict of interest}

The authors hereby declares that there's no conflict of interest and that we all agreed that the paper be published.

\section{References}

[1] Eklof B, Rutherford RB, Bergon JJ, et al. Revision of the CEAP classification for chronic venouse disorders; consensus statement. J. Vasc Surg. 2004; 40: 1248-52.

[2] Bergan JJ, Schmid-Schonbein GW, Coleridge-Smith PD, Nicolaides AN, Boisseau MR, Eklof B. Chronic venous disease. N Engl J med. 2006; 355: 488-98.

[3] Eklof B, Rutherford RB Bergon JJ, et al. Revision of the CEAP classification for chronic venouse disorders: consensus statement, Journal of Vascular Surgery. 2004; 40(6): 1248-1252.

[4] Gloviczki P, Comerota AJ, Dalsing MC, et al. The care of patients with varicose veins and associated chronic venous disease: clinical practice guidelines of the Society for Vascular Surgery. 2011; 53(5): 2S-48S.

[5] Rajeev K, Rupin S. Varicocele and male infertility: current status. The journal of Obstetrics and Gynecology of India. November/ December 2005; 55(6): 505- 516.

[6] Chen QM, et al. (Vasodilating action of methylhesperidin). Zhongguo yao lixue bao, Acta Pharmacological Sinica. 1987; 8(4): 344-8. 
[7] Kikuchi K, et al. The potentitation of the coronary dialating and the cardiac action of adenosine) or adenine nucleotides by methylhesperidin, Japanese Journal of Pharmacology. 1986; 16(2): 224-5.

[8] United States Pharmacopoeia-37, National Pharmulary-32, Pharmacopieal forum. 2014; 37: 3820-3822.

[9] Jones L, Braithwaite BD, Selwyn D, Cooke S, Earnshaw J. Neovascularisation in the principal cause of varicose vein recurrence: results of a randomized trial of stripping the long saphenous vein. European journal of vascular and endovascular surgery. 1996; 12(4): 442-5.

[10] Krewson CF, Naghski J. some physical properties of rutine, Journal of the American Pharmaceutical Association. 1952; 41(11): 582-7.

[11] Ulbricht C, Tiffany N, Boon H, Ulbricht C, Basch E, Bent S, Barrette E, Smith M, Sollars D, Dennehy C and Szapary P, Horse Chestnut. Journal of Herbal Pharmacotherapy. 2002; 2(1): 71-85.

[12] Mor D, Dhande P. Varicose vein: An overview of current and herbal Treatments IJPSR. 2017; 8(5): 1959-1966.

[13] Tiwari S, Gehlot S, Gambhir IS. Centella asiatica: A concise drug review with probable clinical uses. Journal of Stress Physiology and Biochemistry. 2011; 7(1): 38-44.

[14] Gohil K, Patel J, Gajjar A. Pharmacological review on Centella asiatica: A potential herbal cure-all. Indian Journal of Pharmaceutical Sciences. 2010; 72(5): 546.

[15] Jäger K, Eichlisberger R, Jeanneret C and Lobs K: Pharmacodynamic Effects of Ruscus Extract (Cyclo 3 Fort) on Superficial and Deep Veins in Patients with Primary Varicose Veins. Clinical Drug Investigation. 1999; 17(4): 265273.

[16] Kumar M. Scope of Ayurvedic Drugs and Leech Therapy in Management of Varicose Vein of Lower Limb. Journal of Biological and Scientific Opinion. 2014; 2(1): 121-123.

[17] Dasaroju S, Gottumukkala KM. Current Trends in the Research of Emblica officinalis (Amla): A Pharmacological Perspective. International Journal of Pharmaceutical Science Review Research. 2014; 24(2): 150-159.

[18] Yadav V, Duvey B, Sharma S, Devi B. Amla (Emblica officinalis)- Medicinal Food and Pharmacological Activity. International Journal of Pharmaceutical and Chemical Sciences. 2014; 3.

[19] Bharambe S V, Darekar A B, Saudagar R B : Emblica officinalis - The wonder of ayurvedic medicine. World Journal of Pharmacy and Pharmaceutical Sciences. 2014; 3(1): 285-306.

[20] Bhowmik D, Kumar S, Paswan S, Srivastava S. Tomato- A Natural Medicine and Its Health Benefits. Journal of Pharmacognosy and Phytochemistry. 2012; 1(1).

[21] Burton-Freeman B, Reimers K. Tomato Consumption and Health: Emerging Benefits, American Journal of Lifestyle Medicine. 2010; 5(2): 182-191.

[22] Francesco Serio, Ayala 0, Bonasia A. Pietro Santamaria: Antioxidant Properties and Health Benefits of Tomato, In book: Search for Natural Drugs, Vol. 13 of the Series Recent Progress in Medicinal Plants, Chapter: 7, Publisher: Studium press, LLC, Houston, Texas (USA). 2006; 13: 163-183.

[23] Fine AM. Oligomeric proanthocyanidin complexes: history, structure, and phytopharmaceutical applications. Alternative Medicine Review, US National Library of Medicine National Institutes of Health. 2000; 5(2): 144-51.

[24] Aslin SA. Role of Citrus Fruits in Health, Journal of Pharmaceutical Science \& Research. 2014; 6(2): 121-123.

[25] Iriz E, Vural C, Ereren E, Poyraz A, Erer D, Oktar L, Gokgoz L, Halit V, Soncul H. Effects of calcium dobesilate and diosmin-hesperidin on apoptosis of venous wall in primary varicose veins. Vasa. 2008; 37(3): 233-40.

[26] Devi PM, Siril EA. Pharmacognostic Studies on Indian Madder (Rubia cordifolia L.). Journal of Pharmacognosy and Phytochemistry. 2012; 1(5).

[27] Devi PM, Siril EA. Traditional and Modern Use of Indian Madder (Rubia cordifolia L.): An Overview. Int. J. Pharm. Sci. Rev. Res. 2014; 25(1): 154-164.

[28] Jagadeesh GA, Emmi SV, Singh R, Rajput N. Ayurvedic Management of Varicose Vein and Varicose Ulcer. Int. J. Res. Ayurveda Pharm. 2015; 6(2).

[29] Dubey SK, Materia medica part 1, 1998; (3) : 51-55, 189-193, 193-195, 196-199, 226-229, $325-331$. 